\title{
Domain rotation induced strain effect on the magnetic and magneto-electric response
} in $\mathrm{CoFe2O} 4 / \mathrm{Pb}(\mathrm{Mg}, \mathrm{Nb}) \mathrm{O} 3-\mathrm{PbTiO} 3$ heterostructures

Zhiguang Wang, Ravindranath Viswan, Bolin Hu, Jie-Fang Li, V. G. Harris, and D. Viehland

Citation: Journal of Applied Physics 111, 034108 (2012); doi: 10.1063/1.3684546

View online: http://dx.doi.org/10.1063/1.3684546

View Table of Contents: http://scitation.aip.org/content/aip/journal/jap/111/3?ver=pdfcov

Published by the AIP Publishing

\section{Articles you may be interested in}

Electric field tuning of non-volatile three-state magnetoelectric memory in FeCo-

$\mathrm{NiFe} 2 \mathrm{O} 4 / \mathrm{Pb}(\mathrm{Mg} 1 / 3 \mathrm{Nb} 2 / 3) 0.7 \mathrm{Ti0} .3 \mathrm{O} 3$ heterostructures

Appl. Phys. Lett. 106, 122406 (2015); 10.1063/1.4916569

Time-dependent magnetoelectric effect in $\mathrm{Fe} / \mathrm{Pb}(\mathrm{Mg} 1 / 3 \mathrm{Nb} 2 / 3) 0.7 \mathrm{Ti} 0.3 \mathrm{O} 3$ heterostructure: A ferromagnetic resonance study

Appl. Phys. Lett. 103, 082905 (2013); 10.1063/1.4819220

Piezo-strain induced non-volatile resistance states in (011)-La2/3Sr1/3MnO3/0.7Pb(Mg2/3Nb1/3)O3-0.3PbTiO3 epitaxial heterostructures

Appl. Phys. Lett. 102, 033501 (2013); 10.1063/1.4788723

Multiferroic PbZrxTi1-xO3/Fe3O4 epitaxial sub-micron sized structures

Appl. Phys. Lett. 100, 102903 (2012); 10.1063/1.3692583

Magnetoelectric Co Fe 20 4/Pb ( Zr $0.52 \mathrm{Ti} 0.48$ ) O 3 double-layer thin film prepared by pulsed-laser deposition

Appl. Phys. Lett. 88, 013111 (2006); 10.1063/1.2162262

MIT LINCOLN

LABORATORY CAREERS

Discover the satisfaction of innovation and service

to the nation
- Space Control

- Air \& Missile Defense

- Communications Systems \& Cyber Security

- Intelligence, Surveillance and

Reconnaissance Systems

- Advanced
Electronics
- Tactical Systems
- Homeland
Protection
- Air Traffic Control

LINCOLN LABORATORY

MassachusetTs Institute of TeChNOLOgY

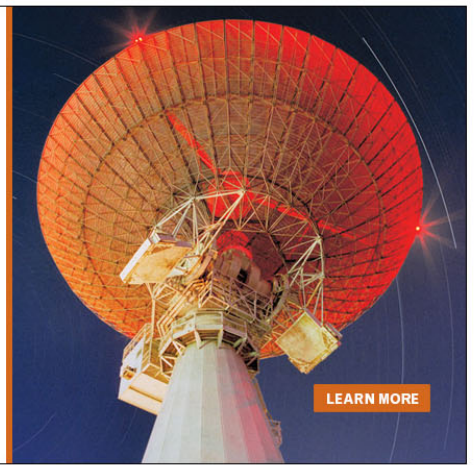




\title{
Domain rotation induced strain effect on the magnetic and magneto-electric response in $\mathrm{CoFe}_{2} \mathrm{O}_{4} / \mathrm{Pb}(\mathrm{Mg}, \mathrm{Nb}) \mathrm{O}_{3}-\mathrm{PbTiO}_{3}$ heterostructures
}

\author{
Zhiguang Wang, ${ }^{1, a)}$ Ravindranath Viswan, ${ }^{1}$ Bolin Hu, ${ }^{2}$ Jie-Fang Li, ${ }^{1}$ V. G. Harris, ${ }^{2}$ and \\ D. Viehland ${ }^{1}$ \\ ${ }^{1}$ Department of Materials Science and Engineering, Virginia Tech, 306 Holden Hall, Blacksburg, \\ Virginia 24061, USA \\ ${ }^{2}$ Department of Electrical and Computer Engineering, Northeastern University, Boston, \\ Massachusetts 02115, USA
}

(Received 2 December 2011; accepted 30 December 2011; published online 10 February 2012)

\begin{abstract}
The present work shows good control of both magnetic and electric properties with electric and magnetic fields, respectively, for epitaxial $\mathrm{CoFe}_{2} \mathrm{O}_{4}$ (CFO) films on $\mathrm{Pb}(\mathrm{Mg}, \mathrm{Nb}) \mathrm{O}_{3}-\mathrm{PbTiO}_{3}$ (PMN-PT). $\mathrm{X}$-ray reciprocal space mapping revealed a transformation between $a$ - and $c$-domains in the PMN-PT under electric field (E). Magnetic hysteresis loop and magnetic force microscopy (MFM) measurements showed a considerable change in the magnetic properties in specific areas of CFO layers poled by MFM probe tips. Furthermore, a pulsed electric field applied to the substrate was found to switch the magnetization of CFO between high and low values, depending on the polarity of E. (C) 2012 American Institute of Physics. [doi:10.1063/1.3684546]
\end{abstract}

\section{INTRODUCTION}

Magnetoelectric (ME) materials represent a vast class of materials encompassing a wide range of crystal structures and phase stabilities. ${ }^{1-5}$ Coupling between ferroelectric (FE) and ferromagnetic (FM) subsystems provides additional degrees of freedom to control either the polarization or magnetization with $\mathrm{H}$ and $\mathrm{E}$, respectively. Although room temperature magnetoelectric effect has been found in a series of single phase materials, such as bulk $\mathrm{Ba}_{3} \mathrm{Co}_{2} \mathrm{Fe}_{24} \mathrm{O}_{41}$ (Ref. 6) and Bi-based double perovskite thin films, ${ }^{7}$ they all have relative complex crystal structure and both FM and FE properties are much weaker than the single functional FE and FM materials. Thus, engineered composite ME materials still promise highest ME coupling effect.

There are three different types of ME composites, based on the phase connectivity between FE and FM phases: (i) These are a (0-3) connectivity where second phase particles are embedded in a primary matrix phase; and (ii) a (2-2) or layer-by-layer one; and (iii) a (1-3) nano-pillar embedded in matrix one. The (0-3) structure promises the highest interfacial area, but the low electrical resistive of the FM phase makes it difficult to fully pole the FE phase, thus reducing the ME coefficient $\left(\alpha_{\mathrm{ME}}\right)$. The $(1-3)$ structure also suffers from leakage due to the existence of low resistance of FM component. Laminate ME composites of magnetostrictive and piezoelectric layers could solve the low resistance problem and have shown much higher values of $\alpha_{\mathrm{ME}}{ }^{8}$ Thin film ME structures are desirable from an applications perspective, facilitating fabrication of non-volatile memories, spintronics, and sensor arrays. Thin film ME structures are composed of FE and FM phases with compatible crystal structures grown on a single crystal substrate. The ME coupling is attributed to the elastic interaction between the two phase layers: thus, the ME effect can be significantly diminished by clamping

${ }^{a)}$ Electronic mail: zgwang@vt.edu. from the substrate. One potential answer to this problem is to deposit epitaxial FM thin films on FE single crystal substrates. The absence of a nonfunctional substrate could help ensure free-expression of large ME coupling effects.

Eerenstein et al. have grown epitaxial $\mathrm{La}_{0.66} \mathrm{Sr}_{0.33} \mathrm{M}-$ $\mathrm{nO}_{3}$ (LSMO) thin films on single crystal $\mathrm{BaTiO}_{3}$ (BTO) substrates and observed giant magnetization changes near a $\mathrm{R} \rightarrow \mathrm{O}$ phase transition. ${ }^{9}$ Yang et al. deposited epitaxial $\mathrm{CoFe}_{2} \mathrm{O}_{4}(\mathrm{CFO})$ thin films on $\mathrm{Pb}(\mathrm{Mg}, \mathrm{Nb}) \mathrm{O}_{3}-\mathrm{PbTiO}_{3}(\mathrm{PMN}-$ $\mathrm{PT})$ substrates to study the magnetization change under different electric fields and observed a converse ME coupling coefficient of $\alpha=\mu_{0} d \mathrm{M} / d \mathrm{E}=3.2 \times 10^{-8} \mathrm{sm}^{-1} .{ }^{10}$ Thiele et al. reported growth of LSMO on PMN-PT substrate and obtained a strain-mediated converse ME coupling coefficient of $\sim 6 \times 10^{-8} \mathrm{sm}^{-1} .{ }^{11}$ However, all of these studies focused on the overall magnetization change due to a strain introduced by an external electric field (E) on the piezoelectric substrate or the switching of magnetization near a phase transition. Local magnetization changes under E are potentially more important for applications.

\section{EXPERIMENT DETAILS}

Here, we deposited epitaxial CFO thin films on single crystal PMN-PT by pulsed laser deposition (PLD) to study the domain rotation induced strain effect on the local magnetic properties of $\mathrm{CFO}$, and the corresponding ME coupling effects. X-ray mesh and line scans were used to analyze domain wall shifts and rotations in the PMN-PT substrate, as well as the strain induced crystal lattice changes of the CFO films. Piezoelectric force (PFM) and magnetic force microscopy (MFM) were used to observe the rotation of the ferroelectric domains in the PMN-PT substrate, and the magnetization contrast change between poled and surrounding areas in the $\mathrm{CFO}$ thin films, respectively. A vibrating sample magnetometer (VSM) was used to quantitatively study the magnetization change in the CFO thin films under 
different strain conditions induced from the piezoelectric substrate. Both direct and converse ME coupling coefficient were measured.

\section{RESULTS AND DISCUSSION}

\section{A. Strain and domain rotation measurements}

Figures 1(a) and 1(b) show the reciprocal space mappings (RSM) of CFO on (001) PMN-PT substrates before and after application of $\mathrm{E}$, respectively. In the unpoled condition, the PMN-PT substrate had a tetragonal (T) structure, and showed a multi-domain ( $a$ - and $c$-) configuration. This domain distribution should be similar for in- and out-ofplane. Accordingly, in Fig. 1(a), $a$ - and $c$-domains can be seen to exhibit similar diffraction intensities due to equal populations.

We then applied $\mathrm{E}=15 \mathrm{kV} / \mathrm{cm}$ along the vertical direction through the substrate, poling the sample and rotating the $a$-domains by $90^{\circ}$ transforming them into $c$-domains. This domain transformation process was clearly evidenced by a decreased $a$-domain peak intensity in Fig. 1(b): The diffraction from the $a$-domains formed a small area with a very weak intensity. This change in the RSM demonstrates, after poling, that most of the domains are rotated to have their polarization axes along the out-of-plane direction. This means that PMN-PT becomes a quasi-single $c$-domain substrate. The crystal lattice parameters of PMN-PT were determined to be $\left(a_{t}, c_{t}\right)=(4.032,4.083)$, where the maximum strain induced by the domain reorientation was $\varepsilon=(a-c) / a=0.0126$. Next, we measured the maximum induced stain under an ac electric field ( $\varepsilon$-E response), as shown in Fig. 1(c). In this case, the maximum induced strain was about 0.006 under an ac electric field of $15 \mathrm{kV} / \mathrm{cm}$.

The induced strain in the PMN-PT substrate was transferred to the epitaxially grown $\mathrm{CFO}$ thin films, thus changing the crystal lattice parameters and spin state of the CFO. Figure 1(d) shows XRD line scans for the CFO/PMN-PT het- erostructures. The PMN-PT substrate had a tetragonal structure, and hence both (002) and (200) zones for CFO were evident. Comparison of these diffraction peaks before and after poling reveals (i) an obvious left-shift of the CFO peaks after poling due to strain from PMN-PT; and (ii) a dramatically decreased intensity for the PMN-PT (200) peak, corresponding to the $a$ - to $c$-domain transformation.

\section{B. PFM and MFM measurements}

Figure 2(a) shows an AFM topography image of the PMN-PT substrate, revealing a roughness of only about $5 \mathrm{~nm}$ after polishing. Figure 2(b) shows a contrast PFM image, where the center square was poled. The surrounding unpoled area reveals stripe-like domains with a width of about $100 \mathrm{~nm}$ that are evenly distributed throughout. The bright and dark contrast areas correspond to regions having different polarization orientations for the vertically aligned $c$-domains. Along (001), we cannot image $a$-domains as PFM does only measure a displacement in the vertical direction. After poling the center area $(5 \mu \mathrm{m} \times 5 \mu \mathrm{m})$ with $\mathrm{E}=5 \mathrm{kV} / \mathrm{cm}$, one can see that the square is entirely dark, demonstrating the presence of a single $c$-domain state. Figure 2(c) shows a AFM topography image of the CFO film, revealing a smooth surface. We then poled the center area $(5 \mu \mathrm{m} \times 5 \mu \mathrm{m})$ of the CFO/PMN-PT heterostructure and obtained MFM images, which revealed obvious contrast differences between poled and unpoled areas. The MFM probe tip was magnetized in the up direction, and the sample was magnetized in the down direction: Thus the higher the outof-plane remnant magnetization, the lighter the contrast. In the center poled area, the transformation to $c$-domains applies a compressive strain to the CFO film. Since CFO has a negative magnetostriction, the magnetic moments would prefer to lay in the in-plane direction under compressive strain, resulting in a decreased remnant magnetization along the out-of-plane direction. The darkened area in the center of
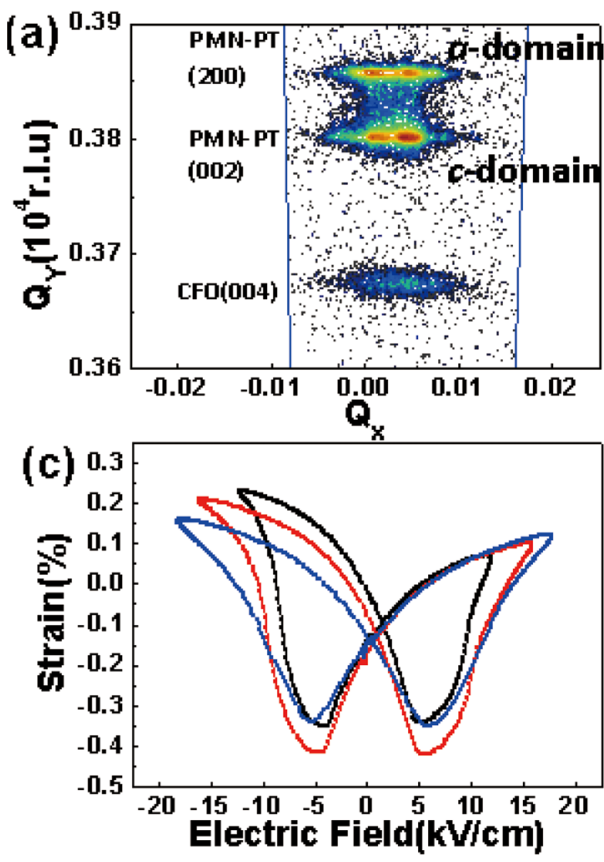
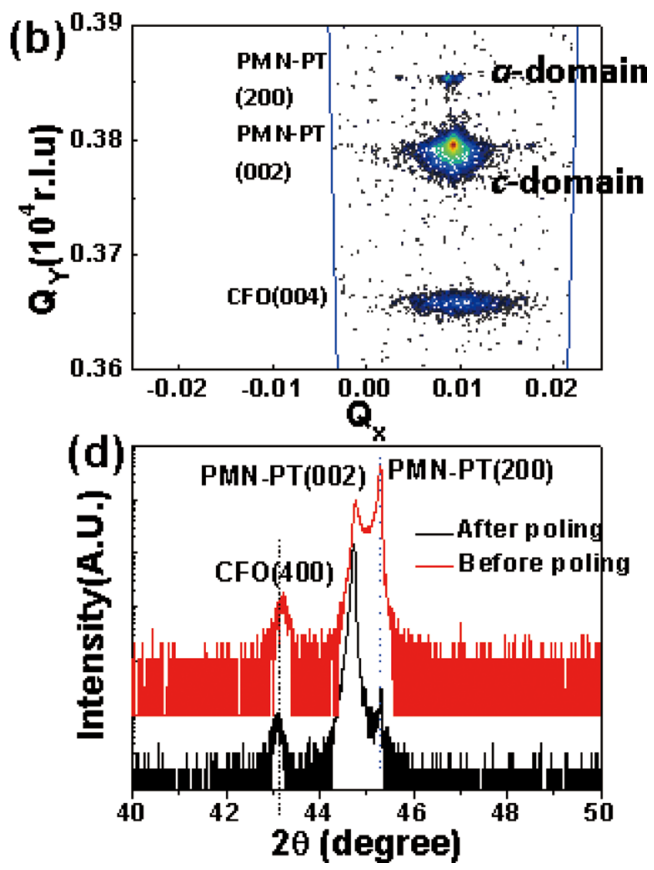

FIG. 1. (Color online) (a) Reciprocal space map of CFO/PMN-PT heterostructure with multi-domain structure; (b) Reciprocal space map of CFO/PMN-PT heterostructure with quasi single $c$-domain structure after poling; (c) strain-electric field curve of piezoelectric PMN-PT substrate; (d) XRD line scans of the CFO/PMN-PT before and after electric field poling. 

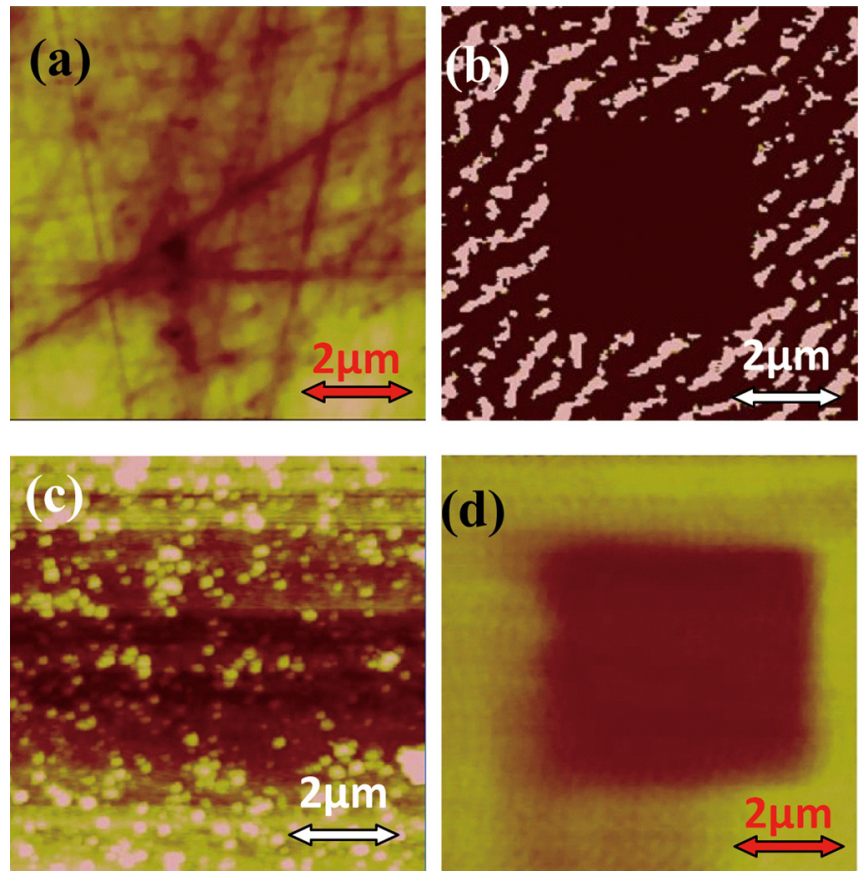

FIG. 2. (Color online) (a) Topographic AFM image of PMN-PT single crystal substrate; (b) PFM measurement of domain rotation in PMN-PT; (c) topographic AFM image of CFO thin film on PMN-PT; (d) MFM measurement of the strain effect on the magnetic response in the poled area.

the MFM image clearly demonstrates this compressive strain induced decrease in the remnant magnetization in the $\mathrm{CFO}$ layer.

\section{Magnetic hysteresis loop and ME coupling measurements}

Quantitative analysis of the strain induced magnetization anisotropy change was then done by VSM measurements. Figure 3(a) shows the in-plane magnetic hysteresis loops of the sample before and after the $a$ - to $c$-domain transformation. This ferroelectric domain transformation in the PMN-PT substrate resulted in a $220 \%$ increase in the remnant magnetization along the in-plane direction. On the contrary, along the out-of-plane direction, the $\mathrm{M}-\mathrm{H}$ loops in Fig. 3(b) exhibited decreased remnant $(67 \%)$ and saturation (72\%) magnetizations after the ferroelectric $a$ - to $c$-domain transformation. This decrease in the remnant magnetization along the out-of-plane direction was also confirmed by the MFM image of Fig. 2(d). We should note that the saturation magnetization should be equal to the spontaneous magnetization $\left(M_{s}\right)$ in a ferromagnet, and that $M_{s}$ can be notably affected by the strain conditions. ${ }^{10,11}$ Thus, the giant strain induced during domain rotation resulted in a dramatic change in both remnant and saturation magnetization values. To eliminate a possible charging effect from the ordering of electric dipoles in the PMN-PT substrate during VSM measurements, we performed similar measurements for a pure PMN-PT substrate without a deposited CFO layer: no magnetization signal was found for both multi-domain and single-domain PMN-PT.

Next, we fixed the sample on the VSM holder and applied an electric field during the magnetization-time measurement. The converse ME coefficient of the CFO/PMN-PT heterostructures was then measured, given in Fig. 3(c). Square wave $50 \mathrm{~V}$ pulses $(\mathrm{E}=1 \mathrm{kV} / \mathrm{cm})$ were applied across the PMN-PT substrate, and a change in magnetization of $\Delta \mathrm{M} \approx 0.1 \mathrm{mEMU}$ was measured. The converse ME coefficient $\alpha_{M E}$ $=\mu_{0}(\partial M / \partial E) H$ was then calculated to be $\alpha_{\mathrm{ME}}=1 \times 10^{-7}$ $\mathrm{sm}^{-1}$ for the CFO layers. This is a much higher value than $\alpha_{\mathrm{ME}}=2.3 \times 10^{-8} \mathrm{sm}^{-1}$ previously reported for LSMO films on $\mathrm{BaTiO}_{3}$ single crystals near an induced $\mathrm{R} \rightarrow \mathrm{O}$ transformation. ${ }^{9}$ Our measured value is comparable with that for bulk laminate $\mathrm{ME}$ composites $\left(4 \times 10^{-7} \mathrm{sm}^{-1}\right){ }^{12}$ More importantly, please note that our giant converse ME effect was achieved by means of a reversible electric field and not via a phase transition at a particular temperature.

Finally, we performed direct magneto-electric measurements of $\alpha_{\mathrm{ME}}$, as shown in Fig. 3(d). Details of the measurement method were reported previously. ${ }^{13}$ A magnet was
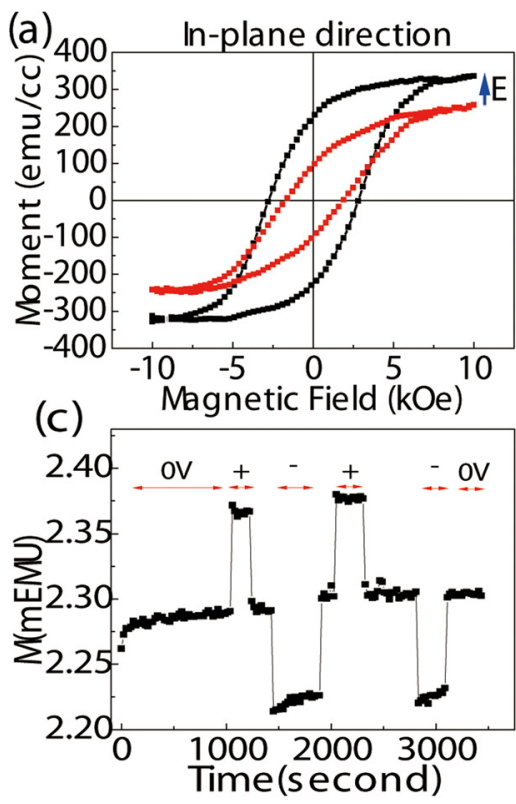
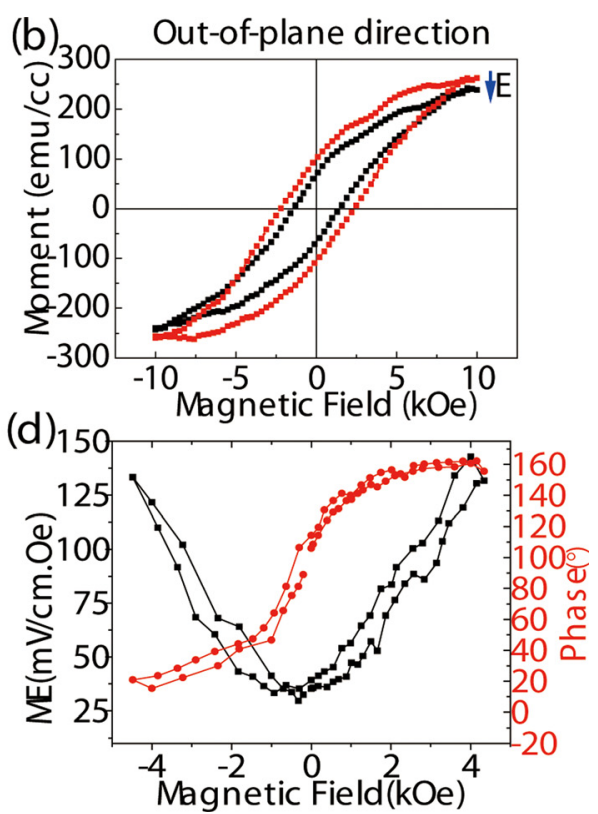

FIG. 3. (Color online) (a),(b) Magnetic hysteresis loops of CFO thin films with different domain configuration in the in-plane direction and out-ofplane directions, respectively; (c) magnetization step change under square wave electric voltage $( \pm 50 \mathrm{~V})$; and (d) voltage ME coupling coefficient curve with phase change inset. 
used as the dc magnetic bias field $\left(\mathrm{H}_{\mathrm{dc}}\right)$. A $10 \mathrm{kHz}$ ac magnetic field $\mathrm{H}_{\mathrm{ac}}$ was then applied along the long axis of the heterostructure. This resulted in an induced strain, via magnetostriction, along the CFO thin film. The induced strain was then transmitted to the PMN-PT substrate, resulting in an output voltage that was detected by a lock-in amplifier. Figure 3(d) shows $\alpha_{\mathrm{ME}}$ as a function of $\mathrm{H}_{\mathrm{dc}}$. A maximum value of $\alpha_{\mathrm{ME}} \approx 140 \mathrm{mV} / \mathrm{cm}$-Oe was found. The shape of the ME coupling coefficient curve is different from that of the NFO/PZT (Ref. 8) bulk ceramic materials that exhibited a saturation of $\alpha_{\mathrm{ME}}$ near 1000e, above which point $\alpha_{\mathrm{ME}}$ decreased with further increasing $\mathrm{H}_{\mathrm{dc}}$. It is well known that the magnetization is determined by magnetic domains, and the domain size and configuration are notably influenced by the stress state of the material: ${ }^{14,15}$ Thus, strained CFO epitaxial thin films may require a higher applied $\mathrm{H}_{\mathrm{dc}}$ to reach saturation value than bulk ceramics. We can also measure the phase difference between the ac source signal and the output voltage that indicates the directions of the ac magnetic field and the corresponding changing magnetostriction direction, using lock-in measurement system. The maximum phase change could reach $150^{\circ}$, as shown in the inset of Fig. 3(d), which indicates that the magnetostriction and ac magnetic field are in the same direction at $-4 \mathrm{kOe}$ magnetic field, whereas they should change in the opposite direction when we increase the magnetic field to $4 \mathrm{kOe}$. It is very difficult to measure the ME effect of thin film structures due to the clamping effect, but in our CFO/PMN-PT structure all the magnetostriction changing could be collected by the piezoelectric substrate, thus we overcame the clamping effect. Also, we have carried similar measurement for PMN-PT substrates without CFO, and no voltage signal is detected except for very small random noise. All these measurements confirm that the output voltage is the real ME coupling signal but not a signal due to the electromotive force effect.

\section{CONCLUSIONS}

In summary, we have grown epitaxial CFO thin films on PMN-PT substrates. PFM images revealed domain control in precise positions under an external electric field. The strain effect on the magnetic properties of CFO thin films was then measured by MFM, which showed the possibility of writing on CFO thin films with an electric field by a MFM probe tip. This induced strain could affect both magnetic anisotropy and spontaneous magnetization of the CFO thin films, which are demonstrated by the dramatic difference in both remnant and saturation magnetization between the original multidomain structure with poled single-domain samples. Finally, both converse and direct ME measurement showed giant ME coupling effects.

\section{ACKNOWLEDGMENTS}

We would like to gratefully acknowledge financial support from the U.S. Department of Energy under Contract No. DE-AC02-98CH10886 and the Office of the Air-Force Office of Scientific Research under Grant No. FA9550-09-10552.

${ }^{1}$ H. Zheng, J. Wang, S. E. Lofland, Z. Ma, L. Mohaddes-Ardabili, T. Zhao, L. Salamanca-Riba, S. R. Shinde, S. B. Ogale, F. Bai, D. Viehland, Y. Jia, D. G. Gchlom, M. Wuttig, A. Roytburd, and R. Ramesh, Science 303, 661 (2004).

${ }^{2}$ F. Zavaliche, T. Zhao, H. Zheng, F. Straub, M. P. Cruz, P.-L. Yang, D. Hao, and R. Ramesh, Nano Lett. 7, 1586 (2007).

${ }^{3}$ H. Zheng, F. Straub, Q. Zhan, P. L. Yang, W. K. Hsieh, F. Zavaliche, Y. H. Chu, U. Dahmen, and R. Ramesh, Adv. Mater. 18, 2747 (2006).

${ }^{4}$ L. Yan, Z. P. Xing, Z. G. Wang, T. Wang, G. Y. Lie, J. F. Li, and D. Viehland, Appl. Phys. Lett. 94, 192902 (2009).

${ }^{5}$ Z. Wang, Y. Yang, R. Viswan, J. Li, and D. Viehland, Appl. Phys. Lett. 99, 043110 (2011).

${ }^{6}$ Y. Kitagawa, Y. Hiraoka, T. Honda, T. Ishikura, H. Nakamura, and T. Kimura, Nature Mater. 9, 797 (2010).

${ }^{7}$ R. Nechache, C. V. Cojocaru, C. Harnagea, C. Nauenheim, M. Nicklaus, A. Ruediger, F. Rosei, and A. Pignolet, Adv. Mater. 23, 1724 (2011).

${ }^{8}$ J. Zhai, Z. Xing, S. Dong, J. Li, and D. Viehland, J. Am. Ceram. Soc. 91, 351 (2008).

${ }^{9}$ W. Eerenstein, M. Wiora, J. L. Prieto, J. F. Scott and N. D. Mathur, Nature Mater. 6, 348 (2007).

${ }^{10}$ J. J. Yang, Y. G. Zhao, H. F. Tian, L. B. Luo, H. Y. Zhang, Y. J. He, and H. S. Luo, Appl. Phys. Lett. 94, 212504 (2009).

${ }^{11}$ C. Thiele, K. Dörr, O. Bilani, J. Rödel, and L. Schultz, Phys. Rev. B 75, 054408 (2007).

${ }^{12}$ S. Dong, J. Zhai, J. Li, and D. Viehland, Appl. Phys. Lett. 89, 252904 (2006).

${ }^{13}$ Z. Wang, L. Yan, Y. Yang, J. Li, J. Das, A. Geiler, A. Yang, Y. Chen, V. G. Harris, and D. Viehland, J. Appl. Phys. 109, 034102 (2011).

${ }^{14}$ L. J. McGilly, A. Schilling, and J. M. Gregg, Nano Lett. 10, 4200 (2010).

${ }^{15}$ D. Y. He, L. J. Qiao, Alex A. Volinsky, Y. Bai, and L. Q. Guo, Phys. Rev. B 84, 024101 (2011). 